\title{
Modifications de l'OPAS, de la LA et de la LiMA
}

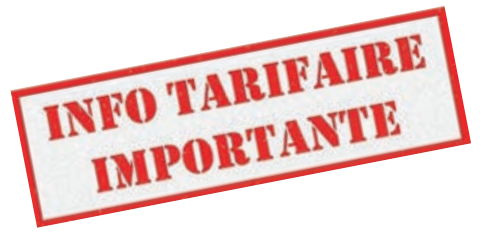

\author{
Susanne Christen ${ }^{a}$, Thomas Kessler ${ }^{b}$ \\ ${ }^{a}$ Dre, $\mathrm{FMH}$, cheffe suppléante de la division Médecine et tarifs ambulatoires; ${ }^{\mathrm{b}} \mathrm{FMH}$, expert, division Médecine et tarifs ambulatoires
}

L'article ci-après présente les principales modifications de l'ordonnance sur les prestations de l'assurance des soins (OPAS), de la liste des analyses (LA) et de la liste des moyens et appareils (LiMA) qui entrent en vigueur aux $1^{\text {er }}$ mars, $1^{\text {er }}$ avril et $1^{\text {er }}$ juillet 2019.

Ordonnance sur les prestations de l'assurance des soins (OPAS): à l'art. 12a let. a, b, d et g OPAS, c'est désormais le Plan de vaccination 2019 qui s'applique. En revanche, le Plan de vaccination 2018 et les recommandations de l'OFSP et de la CFV du 22 octobre 2018 (cf. Bulletin de l'OFSP 43/18) s'appliquent pour la vaccination contre les méningocoques. Pour l'art. 12d let. a OPAS Test VIH, la directive de l'OFSP «Dépistage du VIH effectué sur l'initiative des médecins en présence de certaines pathologies (maladies évocatrices d'une infection à VIH)» du 18 mai 2015 s'applique. A l'art. 12e let. d OPAS Dépistage du cancer du côlon, le canton du Valais est désormais inclus dans les programmes de dépistage pris en charge. A l'art. 13 let. $\mathbf{b}^{\text {ter }}$ OPAS Test prénatal non invasif (TPNI), il convient de tenir compte de l'avis d'experts $n^{\circ} 52$ de Gynécologie suisse du 14 mars 2018. En outre, en cas de grossesse gémellaire, les TPNI par micro-réseau ou par polymorphisme mononucléotidique (SNP) sont exclus de la prise en charge des coûts par l'assurance.

Ordonnance sur les prestations de l'assurance des soins (OPAS), annexe 1: selon le point 1.4 Urologie et proctologie, annexe 1 OPAS, l'électroneuromodulation percutanée du nerf tibial est prise en charge à partir du $1^{\text {er }}$ mars 2019 pour le traitement de l'hyperactivité vésicale idiopathique et de l'incontinence fécale après échec des traitements conservateurs. Au point 2.1 Médecine interne générale, annexe 1 OPAS, les normes «FACT-JACIE International Standards for hematopoietic Cellular Therapy Product Collection, Processing and Administration", $7^{\mathrm{e}}$ édition de mars 2018, s'appliquent pour la greffe de cellules souches hématopoïétiques à partir du $1^{\mathrm{er}}$ mars 2019. Selon le point 2.3 Neurologie, $y$ compris la thérapie des douleurs et l'anesthésie, annexe 1 OPAS, le traitement par ultrasons focalisés du palli- dum, du thalamus et du noyau subthalamique est en évaluation jusqu'au 30 juin 2020 en cas de diagnostic établi d'une maladie de Parkinson idiopathique, avec une progression des symptômes sur un minimum de deux ans et un contrôle insuffisant des symptômes par le traitement dopaminergique (phénomène off, fluctuations on/off, dyskinésies on). Selon le point $5 \mathrm{Der}$ matologie, annexe 1 OPAS, l'utilisation des équivalents de peau est prise en charge à certaines conditions à partir du $1^{\text {er }}$ mars 2019 pour le traitement des plaies chroniques. Au point 9 Radiologie, annexe 1 OPAS, différentes modifications concernent la tomographie par émission de positrons (TEP, TEP/TC); elle est p. ex. prise en charge à partir du $1^{\text {er }}$ mars 2019 en cardiologie, en cas de suspicion d'infection d'implants cardiologiques. Il en va de même en médecine interne et en infectiologie, p. ex. en cas de fièvre d'origine inconnue après un examen non conclusif, en cas de suspicion d'infection de greffons vasculaires et en cas d'échinococcose alvéolaire dans la perspective d'une éventuelle suspension du traitement médicamenteux. Enfin, la tomographie est en cours d'évaluation pour la localisation préopératoire d'un adénome parathyroïdien en cas d'hyperparathyroïdie primaire, si l'imagerie médicale conventionnelle est négative ou non conclusive (scintigraphie au sestamibi ou -TEMP/TC).

Vous trouverez le détail des modifications de l'OPAS (y compris annexe 1) sur le site Internet de l'Office fédéral de la santé publique (OFSP): https://www.bag. admin.ch/bag/fr/home.html $\rightarrow$ Lois \& autorisations $\rightarrow$ Législation $\rightarrow$ Assurances $\rightarrow$ Bases légales Assurancemaladie $\rightarrow$ Loi fédérale sur l'assurance-maladie $\rightarrow$ Modifications de l'ordonnance sur les prestations de l'assurance des soins (OPAS) 


\section{Modifications de la liste des analyses (LA) au $1^{\text {er }}$ mars 2019}

Pour le laboratoire au cabinet, le supplément $\mathbf{4 7 0 7 . 1 0}$ pour chaque analyse présentant le suffixe $C$ est maintenu pour les analyses du chiffre 5.1.2.2.2 Analyses complémentaires et les analyses du chiffre 5.1.3 Analyses pour les médecins avec certains titres postgrades; en revanche, il est supprimé pour les analyses du chiffre 5.1.4 Analyses effectuées par les médecins dans le cadre d'une consultation à domicile.

L'analyse 1735.00 Troponine, T ou I, test rapide avait été supprimée au $1^{\mathrm{er}}$ septembre 2018 sauf pour le point 5.1.4 Analyses effectuées par les médecins dans le cadre d'une consultation à domicile. Désormais, elle est aussi supprimée pour les consultations à domicile, mais peut être remplacée par la position 1734.01 Troponine, $T$ ou I, qn, par immunoassay. Pour cela, les limitations suivantes sont à observer: 1) pas par tests rapides (détermination qualitative); 2) non cumulable avec la position 1249.01 Créatine-kinase (CK), total; 3) la prise en charge des coûts de l'analyse est limitée au 30 juin 2021.

Vous trouverez le détail des modifications de la liste des analyses sur le site Internet de l'Office fédéral de la santé publique (OFSP): www.bag.admin.ch/la

\section{Modifications de la liste des moyens et appareils (LiMA) au $1^{\text {er }}$ avril 2019}

Au point 4.6, les notions de "par an" et "prorata" ont été définies plus précisément:

Un traitement, et plus exactement l'achat de produits thérapeutiques, ne commence pas nécessairement au $1^{\mathrm{er}}$ janvier d'une année. Le versement d'un montant maximal de remboursement (MMR) "par an (prorata)» se réfère toujours à la portion de l'année civile durant laquelle les produits ont été effectivement utilisés.

Correspondance: FMH / division Médecine et tarifs ambulatoires

Baslerstrasse 47 CH-4600 Olten

Tél. 0313591230

Fax 0313591238

tarife.ambulant[at]fmh.ch traitement est poursuivi et un montant maximal de CHF 400.- pourra être remboursé pour l'année civile. En règle générale, le remboursement est arrondi à des nombres entiers, par exemple lorsqu'un produit doit être renouvelé toutes les 2 semaines.

Un MMR «par an» est généralement utilisé pour des produits ou des aides qui ne sont pas achetés plusieurs fois par an ou dont l'utilisation est irrégulière. Il comprend le remboursement maximal possible pour les 365 jours suivant le premier achat. C'est-à-dire non pas par année civile mais par année de traitement en cours.

Un exemple: les verres de lunettes/lentilles de contact sont remboursés via un MMR jusqu'à l'âge de 18 ans au maximum une fois par an. Si le premier achat a eu lieu le 31 octobre 2015, l'achat suivant ne pourra être réalisé au plus tôt qu'en novembre 2016.

De manière exceptionnelle, du matériel acheté plusieurs fois par an mais utilisé de manière irrégulière peut également bénéficier d'un MMR par an. Les bandelettes de mesure de la glycémie chez les patients diabétiques non-insulinorequérants en sont un exemple. Dans ce cas, une utilisation une fois par jour (correspondant à un remboursement au prorata) n'est pertinente que dans des cas exceptionnels. Cela permet aussi de tenir compte de périodes courtes avec des mesures plus intensives, par exemple en cas de changement de traitement. De la même manière, seul un petit emballage de bandelettes peut être remboursé en cas de mesures de la glycémie débutant à la fin d'une année civile.

Enfin, certains chapitres ont subi d'importantes modifications:

15. Aides pour l'incontinence

17. Articles pour traitement compressif (y c. bas de soutien)

29. Matériel de stomathérapie

35. Matériel de pansement

Vous trouverez le détail des modifications de la liste des moyens et appareils (LiMA) sur le site Internet de l'Office fédéral de la santé publique (OFSP): www.bag. admin.ch/lima 